\title{
The Effect of Time on Gait Recognition Performance
}

\author{
Darko S. Matovski, Mark S. Nixon, Sasan Mahmoodi, Member, IEEE, and John N. Carter
}

\begin{abstract}
Many studies have shown that it is possible to recognize people by the way they walk. However, there are a number of covariate factors that affect recognition performance. The time between capturing the gallery and the probe has been reported to affect recognition the most. To date, no study has isolated the effect of time, irrespective of other covariates. Here, we present the first principled study that examines the effect of elapsed time on gait recognition. Using empirical evidence we show for the first time that elapsed time does not affect recognition significantly in the short-medium term. This finding challenges the existing view in the literature that time significantly affects gait recognition. We employ existing gait representations on a novel dataset captured specifically for this study. By controlling the clothing worn by the subjects and the environment, a Correct Classification Rate (CCR) of $95 \%$ has been achieved over the longest time period yet considered for gait on the largest ever temporal dataset. Our results show that gait can be used as a reliable biometric over time and at distance if we were able to control all other factors such as clothing, footwear etc. We have also investigated the effect of different type of clothes, variations in speed and footwear on the recognition performance. The purpose of these experiments is to provide an indication of why previous studies (employing the same techniques as this study) have achieved significantly lower recognition performance over time. Our experimental results show that clothing and other covariates have been confused with elapsed time previously in the literature. We have demonstrated that clothing drastically affects the recognition performance regardless of elapsed time and significantly more than any of the other covariates that we have considered here.
\end{abstract}

Index Terms-Aging, elapsed time, gait biometrics, gait recognition, object recognition, personal identification, time.

\section{INTRODUCTION}

$\mathbf{T}$ HE way we walk, combined with our posture, is known as gait. Many studies have demonstrated that gait has the potential to become a powerful biometric for surveillance and access control [1]-[3]. There is a continuous growth in the number of surveillance systems in efforts to improve safety and security. These systems have yet to include recognition capability. The major advantages of gait are: noninvasive, hard to conceal and capable of being acquired at a distance. Gait can be detected at a very low resolution and therefore it has a unique advantage over all other biometrics for visual surveillance systems. Re-

Manuscript received January 21, 2011; revised October 10, 2011; accepted October 13, 2011. Date of publication November 15, 2011; date of current version March 08, 2012. This work was supported by the National Physical Laboratory (NPL UK). The associate editor coordinating the review of this manuscript and approving it for publication was Dr. Fabio Scotti.

The authors are with the School of Electronics and Computer Science (ISIS Research Group), University of Southampton, SO17 1BJ U.K. (e-mail: dm08r@ecs.soton.ac.uk; msn@ecs.soton.ac.uk; sm3@ecs.soton.ac.uk; jnc@ecs.soton.ac.uk).

Color versions of some of the figures in the paper are available online at http:// ieeexplore.ieee.org.

Digital Object Identifier 10.1109/TIFS.2011.2176118 cently, gait has been used as a forensic tool [4], [5] and there have already been convictions based on the evidence produced by gait analysis. There are a number of situations in which gait is the only biometric available from a crime scene.

However, there are a number of covariates (also known as exploratory variables) that affect gait recognition. Studies quantifying the effect of covariates on the performance of gait recognition algorithms include [6]-[8]. Covariate factors can be related either to the subject itself (e.g., different clothing) or to the environment (e.g., different walking surface). The research in [9] considers five covariates that affect recognition: viewing angle, shoe type, walking surface, carrying objects and elapsed time between sequences being compared. Understanding these factors is crucial to developing robust and accurate gait recognition algorithms. The effect of a particular covariate on the recognition performance depends on the algorithm adopted. There are two main approaches to gait recognition: model-based and model-free. Model-based approaches use the human body structure [10]-[12] and model-free methods employ the whole motion pattern of the human body [13], [14]. In this paper we use existing model-free approaches to examine the effect of time and other covariate factors. We use the Gait Energy Image (GEI) [15] since it is one of the most popular gait representations used so far and we use the Gait Entropy Image (GEnI) [16] as it is a recent method and it is believed to be invariant to changes in covariate factors.

There is a consensus in the literature that the time taken between recording the gallery and the probe affects recognition performance the most [9]. Time as a covariate has not been considered explicitly in many studies mainly due to the lack of suitable database. Since gait is a behavioral biometric, an important question arises: "Is it possible to recognize someone reliably after a certain period of time has elapsed?" Permanence is an important characteristic of any biometric. A biometric trait that changes significantly over time is unlikely to be useful for recognition in numerous scenarios.

In previous studies considering the effect of elapsed time on recognition by gait, in [17] a probe consisting of ten subjects is matched to a gallery containing 115 subjects. The subjects are filmed 6 months apart and a CCR of $37 \%$ is achieved. A study [9] reported a substantial drop in recognition performance (at rank 1) from $78 \%$ to $3 \%$ when shoe and time (6 months) covariates are introduced, using the Gait Challenge database. In [18], a different recognition algorithm is used on the same dataset. Seventy silhouettes are chosen and manually subtracted from the background. The recognition performance over time dropped to $10 \%$. In [10], two different recognition algorithms are employed on the MIT database. Experiments are performed over two months in an indoor environment with different backgrounds and lighting. On a dataset of 24 subjects, the recognition rate varies between $30 \%-60 \%$. Another study [19] using the 
MIT database shows a performance drop to $45 \%$ from $100 \%$ for samples taken on the same day. Experiments on the UMD database reveals a drop in recognition performance to $30 \%$ over 3 months using 25 subjects in indoor environment [20]. Clothing was not controlled in any of these studies.

Many studies, such as [6], [7] and [21], do not consider temporal data and focus solely on data acquired on the same day.

Papers such as [22] treat time (over 6 months) as the most difficult covariate for gait recognition. They have proposed a new pattern classification method to solve the elapsed time problem in gait recognition.

In this paper, we present empirical evidence to show that by controlling clothing worn by the subjects and the environment the recognition performance is not affected drastically over 9 months. This is the longest time period yet considered for gait and we report, rather to our surprise, a much higher recognition rate than any previous study. We employ existing gait representations to examine the effects of time and other covariates.

The work presented in this paper does not aim to propose a new recognition algorithm, but builds on our previous work [23], and we have extended the database, the techniques, the analyses and the results to show that elapsed time does not appear to affect the recognition performance considerably in the short-medium term as long as some factors (like clothing, footwear etc.) are kept unchanged. Our work aims to direct the research community towards solving other gait covariates that appear to affect performance more considerably than elapsed time. Consequently, we provide evidence to show that clothing seems to be the major problem for model-free approaches. Studies such as [24] and [25] have recently started to approach this problem. We have used existing gait representations to obtain our results. Lack of a suitable database has been a major obstacle for understanding the effects of time. Our novel database enables researchers to examine each covariate separately and discover new algorithms invariant to each covariate. We show that the ability to recognize someone from a large distance, at a low resolution is not affected by elapsed time if all other characteristics are controlled (even though this is very unlikely to be achieved in the real world). We also consider the effect of walking speed and change of shoes. These experiments are performed using a relatively small number of subjects and samples. Therefore, it is important to note that the primary aim of these experiments is to provide an indication of whether change in any of these is likely to have a significant impact on recognition and not to quantify the exact effect on the performance.

The remainder of this paper is organized as follows: Section II describes existing temporal datasets and describes our new multimodal temporal dataset. Section III elaborates on the methodology used to obtain the results which are presented in Section IV. Finally, the conclusions are presented in Section V.

\section{DESCRIPTION OF DATASETS}

\section{A. Existing Temporal Datasets}

Table I shows some of the most well-known datasets that contain data sampled at different times. Our new database is shown
TABLE I

SumMary of EXISTING TEMPORAL GAIT DATASETS

\begin{tabular}{|c|c|c|c|c|c|}
\hline Name & $\begin{array}{c}\text { No. of } \\
\text { Subjects }\end{array}$ & $\begin{array}{c}\text { No. of } \\
\text { Samples }\end{array}$ & $\begin{array}{c}\text { Time } \\
\text { Difference }\end{array}$ & Views & $\begin{array}{l}\text { Indoor/ } \\
\text { Outdoor }\end{array}$ \\
\hline MIT 2001 [10] & 13 & 194 & 3 months & 1 & $\mathrm{Y} / \mathrm{N}$ \\
\hline HumanID [9] & 33 & 132 & 6 months & 2 & N/Y \\
\hline $\begin{array}{c}\text { SOTON } 2002 \\
{[17]}\end{array}$ & 10 & $\approx 100$ & 7 months & 1 & $\mathrm{Y} / \mathrm{N}$ \\
\hline UMD [20] & 25 & 100 & 3 months & 4 & N/Y \\
\hline $\begin{array}{c}\text { SOTON } \\
\text { Temporal [26] }\end{array}$ & 25 & 2280 & $\begin{array}{c}0,1,3,4,5,8,9 \\
12 \text { months }\end{array}$ & 12 & $\mathbf{Y} / \mathbf{N}$ \\
\hline
\end{tabular}

at the bottom of the table. It is the largest gait temporal dataset with the longest time period yet considered for gait recognition.

Other databases add more than one covariate at a time and therefore it is difficult to quantify the individual effect. Our database allows gait analyses with covariates such as elapsed time, change of clothing, variations in walking speed and the effect of footwear by introducing only a single covariate between two data capture sessions. Such analyses are not possible with any of the existing datasets. For instance in the Gait Challenge dataset, three covariates are added simultaneously: clothes, shoes and time. The UMD database uses different viewing angle for the data recorded in March compared to the data recorded in June. Clothing, shoes and background have not been controlled either. None of the existing databases contains samples over a time period of subjects wearing the same clothes and minimizing as much as possible the effect of other covariates. This is a key difference between our new database and all the existing ones.

As such, investigating the effect of time in a principled manner is not possible with any previous dataset. It is impossible to determine whether the recognition performance varies due to changes of gait over time or due to other factors such as change in clothes, shoes, background or viewing angle. Another advantage of our database is the availability of more than just two acquisition sessions. It is possible to achieve analysis between ten different time periods.

\section{B. Details of Our Novel Dataset}

The data is captured using the University of Southampton Multi-Biometric Tunnel. The "tunnel" is a constrained environment that contains 12 synchronized cameras to capture subject's gait, a camera to capture face video and a camera to take ear images. The intended use of the "tunnel" is at airports and other high throughput environments. It also allows a researcher to perform analysis in a controlled environment and therefore facilitates the deployment of gait in outdoor surveillance/forensic scenarios. The data is acquired automatically in a noninvasive manner as the subject walks through it [26].

A novel and unique database (SOTON temporal) has been created that enables analyses to be performed over time while controlling covariates that are known to affect gait recognition performance. Some of the factors that have been accounted for and remained unchanged over time are as follows.

1) Environment: Background, lighting, walking surface, position of cameras (automatically calibrated)

2) Subjects: No major changes in any subject's life style were reported. None of the subjects reported any major injuries. 

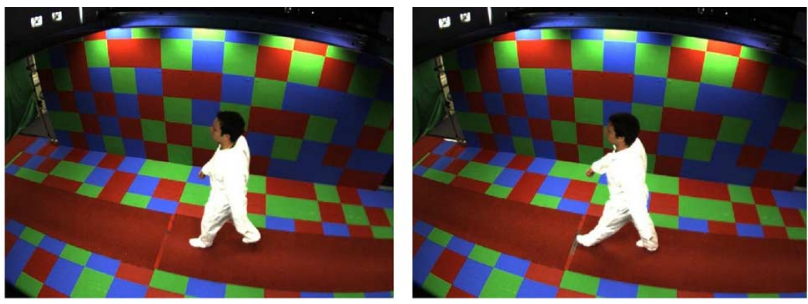

a)
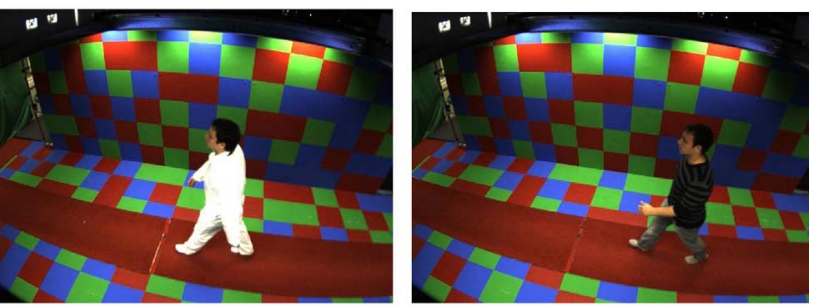

b)

Fig. 1. Raw data frames from SOTON multimodal temporal database. (a) Examples of data taken in sessions 1,2 and 3. (b) Examples of data taken in session 4 (month 9).

\begin{tabular}{|c|c|c|c|c|c|}
\hline Session & $\begin{array}{c}\text { August } \\
(\mathrm{t}=0)\end{array}$ & $\begin{array}{c}\text { Sept } \\
(\mathrm{t}=1)\end{array}$ & $\begin{array}{c}\text { Dec } \\
(\mathrm{t}=4)\end{array}$ & $\begin{array}{c}\text { May } \\
(\mathrm{t}=9)\end{array}$ & $\begin{array}{c}\text { August } \\
(\mathrm{t}=12)\end{array}$ \\
\hline No of subjects & 25 & 23 & 22 & 21 & 18 \\
\hline
\end{tabular}

Fig. 2. Number of subjects for each acquisition session (time is in months).

3) Other: Twenty samples per subject per session were collected to enable samples of similar speeds to be compared without explicitly controlling it.

Twenty-five subjects ( 17 male and 8 female) took part in our study. The age distribution is between $20-52$ years old. Data was acquired in five sessions spanning 12 months. The same subjects were used in all sessions. The number of subjects available for each session is shown in Fig. 2.

The sessions taken in months 0,1 and 4 consist of 20 samples per subject where subjects wear white overalls over their normal clothes. The session in month 9 differs from the previous three. It consists of ten samples of each subject wearing normal clothes in addition to ten samples of each subject wearing overalls. The last session (month 12) was collected to quantify the effect of additional clothing types and footwear. During this session, subjects were asked to wear two different types of "ordinary" clothes that were different from the clothes that they wore in the session recorded in month 9. Twenty-five samples per subject were collected, ten for each type of clothes and additional five of the subjects wearing shoes. The only instruction to subjects is to "walk normally". Examples of raw data frames are shown in Fig. 1.

In hindsight, a session taken in month 7 should also have been collected as it would provide data for differences of 2, 6 and 7 months.

There are 12 cameras available in the Biometric Tunnel, hence a gait sequence is available from 12 different viewing angles.

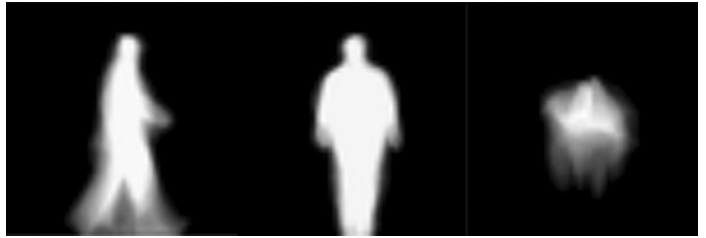

Fig. 3. Example of feature concatenation.

Using our database, it is possible for the first time to isolate the effect of time and other covariates on the performance of gait recognition while keeping other things constant.

\section{Methodology}

The data processing steps are described below.

Step 1-Data acquisition: A data sample consists of 12 video sequences from different angles sampled at $30 \mathrm{f} / \mathrm{s}$. Example of data samples are shown in Fig. 1.

Step 2-Background subtraction: The biometric tunnel uses partially normalized color space combined with a uni-modal Gaussian distribution to model the background. More complex algorithms produce similar results with no significant improvements.

Step 3-Gait-cycle finder: There are at least two gait cycles for each sequence as the subjects walk from the start to the end of the tunnel. By analyzing the silhouettes of a walking subject the most suitable gait cycle is automatically identified.

Step 4-Signatures computation: Two gait signatures are used in this study: Gait Energy Image (GEI) and Gait Entropy Image (GEnI). GEI was chosen because it is a baseline gait signature and is one of the most widely used approaches in the literature. Gait is represented as a single gray scale image obtained by averaging the silhouette extracted over a complete gait cycle. It is computed over a complete gait cycle. If $P(0)_{x, y}$ is the first image of the cycle and the next image is $P(1)_{x, y}$ the average silhouette can be computed using [15]

$$
A S(t)_{x, y}=\frac{1}{t} \sum_{i=0}^{t} P(i)_{x, y}
$$

where $t$ is the total number of images in the gait period.

Three different views of the GEI have been used in our experiments: top view, side view and front view. Two versions of each view have been computed: normalized (Norm) and non-normalized $(N N)$. Unlike the non-normalized signature, the normalized one does not retain the subject's height and body mass. Producing a non-normalized version is possible due to full 3-D reconstruction. The size of the normalized signature is $64 \times 64$ pixels and the size of the non-normalized is $50 \times 50$ pixels.

Gait Entropy Image (GEnI) is a newer approach which captures mostly motion information and is suggested to be robust 


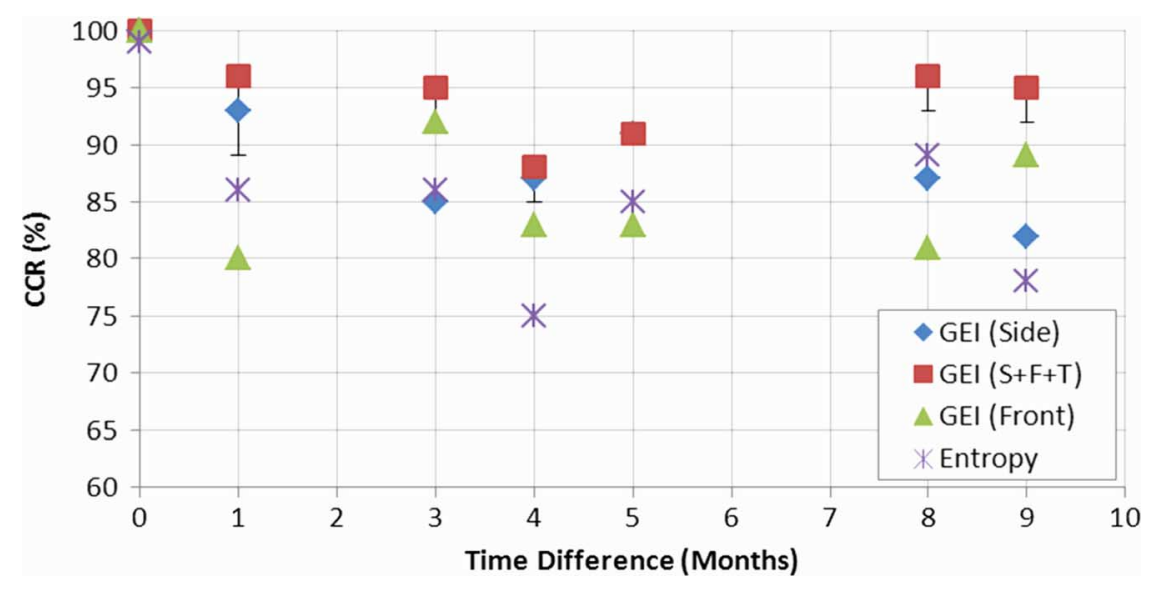

Fig. 4. Recognition performance over time for the GEnI signature, two different views of the GEI signature and fusion of three different GEI views $(\mathrm{S}+\mathrm{F}+\mathrm{T}=$ Fusion of Side, Front and Top view).

to covariate conditions changes. It offers a compact representation and possess the strengths of SVB Frieze Pattern [16]. GEnI is computed by calculating Shannon entropy for every pixel

$$
H(x, y)=-\sum_{k=1}^{K} p_{k}(x, y) \log _{2} p_{k}(x, y) .
$$

$p_{k}(x, y)$ is the probability that a pixel takes on the $k$ th value. A binary image corresponds to $k=2$. GEnI is computed using a binary height normalized image of a walking subject. Consequently, using this signature it is not possible to utilize the benefits of using multiple cameras and producing a non-normalized version is not possible.

Step 5-Feature concatenation: The feature concatenation module creates a modified GEI signature by combining side, front and top view $(S+F+T)$. An example of signature is shown in Fig. 3.

Step 6-Feature Reduction: The feature reduction module reduces the size of the signatures and enables us to perform recognition at different resolutions. The following reduced size signatures have been used: $50 \times 50,40 \times 40,30 \times 30,20 \times 20$, $17 \times 17,15 \times 15,12 \times 12,10 \times 10$ and $7 \times 7$ pixels.

Step 7-Classification: The gait signatures are used directly for classification. Improvements in performance could have been achieved if additional features (e.g., moments) had been extracted from the gait signatures and feature set selection had been performed to understand which moments/features contribute most towards recognition. However, since the focus of this study is not improvement in performance but quantifying the effect of time and other time-dependent covariates these improvements are not deemed necessary.

A distance matrix containing all Euclidian distances between all samples is constructed. The Euclidian distance between two samples is calculated by using the intensity values of the gait signatures. The $k$-Nearest Neighbor classifier $(k-\mathrm{NN})$ was applied using the distance matrix. Experiments for $k=1, k=3$ and $k=5$ are performed.

Step 8-Performance evaluation: The performance evaluation is performed in terms of:
1) Correct Classification Rate (CCR).

2) Receiver operating characteristic (ROC) curve.

3) Equal error rate (EER).

4) Diagram of intra- (within subject) and inter- (between subject) class distributions.

5) The decidability metric $\left(\mathbf{d}^{\prime}\right)$ [27] determines how much overlap there is between two distributions

$$
d^{\prime}=\frac{\left|\mu_{\text {same_subj }}-\mu_{\text {diff_subj }}\right|}{\sqrt{\frac{1}{2}\left(\sigma_{\text {same_subj }}^{2}+\sigma_{\text {diff_subj }}^{2}\right)}}
$$

where $\mu_{\text {same_subj }}$ and $\sigma_{\text {same_subj }}$ refer to the intra-class mean and standard deviation, respectively, and $\mu_{\text {diff_subj }}$ and $\sigma_{\text {diff_subj }}$ refer to the inter-class mean and standard deviation.

The problem becomes more decidable if their means are further apart or their variances are smaller. The $d^{\prime}$ metric should be very similar if changes in experimental conditions do not drastically affect the separation in the feature space.

\section{RESULTS}

\section{A. Effect of Time}

The combinations of probe and gallery used in this experiment are shown in Table II. Each probe and gallery consists of ten samples per subject. In this experiment, we only use the samples of the subjects wearing overalls to provide consistent clothing over time. The non-normalized version of the signature $(50 \times 50$ pixels $)$ is used for this experiment. A 4-fold cross validation is performed and the highest recognition rates are shown in Fig. 4. A 2-fold cross validation is performed for experiments for time differences of 5,8 and 9 months due to a smaller number of samples with overalls filmed in month 9 .

The error-bars for GEI $(S+F+T)$ indicate the lowest results of the 4 -fold cross validation. The error bars for the other signatures are not shown for clarity. The standard deviation for the results of the 4 -fold cross validation is between $0.00-0.03$. Values 
TABLE II

COMBINATION OF PROBE AND GALLERY

\begin{tabular}{ccccccccc}
\hline \hline $\begin{array}{c}\text { Probe } \\
\text { month }\end{array}$ & $\begin{array}{c}\text { Probe } \\
\text { subj. }\end{array}$ & $\begin{array}{c}\text { Gallery } \\
\text { month }\end{array}$ & $\begin{array}{c}\text { Gallery } \\
\text { subj. }\end{array}$ & $\begin{array}{c}\text { EER } \\
\% \text { GEI } \\
\text { Side }\end{array}$ & $\begin{array}{c}\text { EER } \\
\% \\
\text { GEI } \\
\text { Front }\end{array}$ & $\begin{array}{c}\text { d' } \\
\text { GEI } \\
\text { Side }\end{array}$ & $\begin{array}{c}\text { d' } \\
\text { GEI } \\
\text { Front }\end{array}$ & $\begin{array}{c}\text { Time } \\
\text { Diff. } \\
\text { (mont } \\
\text { hs) }\end{array}$ \\
\hline 0 & 25 & 0 & 25 & 4.95 & 3.10 & 2.71 & 2.76 & $\mathbf{0}$ \\
1 & 23 & 1 & 23 & 6 & 4.05 & 2.49 & 2.56 & $\mathbf{0}$ \\
4 & 22 & 4 & 22 & 6.5 & 4.10 & 2.52 & 2.57 & $\mathbf{0}$ \\
1 & 23 & 0 & 25 & 9.35 & 8.61 & 2.14 & 2.12 & $\mathbf{1}$ \\
4 & 22 & 1 & 23 & 10.19 & 7.30 & 2.15 & 2.20 & $\mathbf{3}$ \\
4 & 22 & 0 & 25 & 9.72 & 9.43 & 2.10 & 2.20 & $\mathbf{4}$ \\
9 & 21 & 4 & 22 & 10.00 & 10.61 & 2.24 & 2.12 & $\mathbf{5}$ \\
9 & 21 & 1 & 23 & 9.40 & 9.50 & 2.18 & 2.15 & $\mathbf{8}$ \\
9 & 21 & 0 & 25 & 11.54 & 7.31 & 2.11 & 2.22 & $\mathbf{9}$ \\
\hline \hline
\end{tabular}

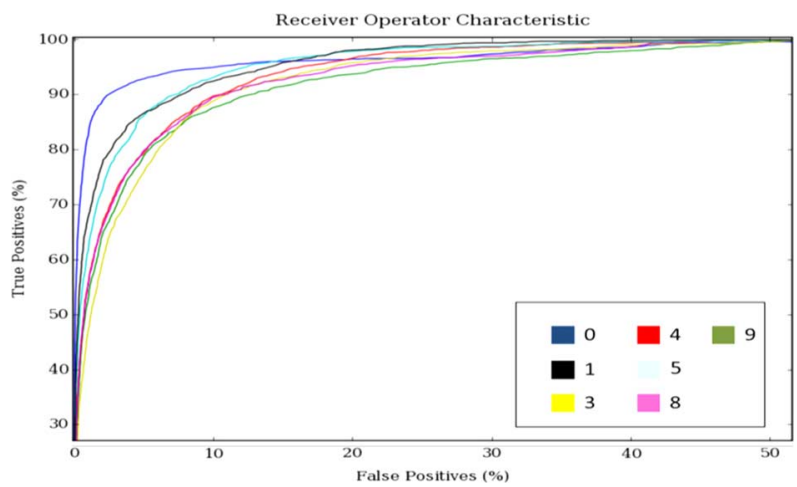

Fig. 5. ROC curve for all combinations of gallery and probe as shown in Table II.

for the EER and the decidability metric $\left(d^{\prime}\right)$ are also shown in Table II.

The results in Fig. 4 clearly indicate that the CCR does not fall considerably over time for any of the signatures considered in this study. The variation of performance over time is similar for all signatures considered. The EER and decidability $\left(d^{\prime}\right)$ for GEI (side) and GEI (Front) shown in Table II indicate that the signatures have a similar discriminatory ability, although the front view performs slightly better in most cases. However, our results show that there is a benefit of combining multiple views to construct a single gait signature. The GEI $(\mathrm{S}+\mathrm{F}+\mathrm{T})$ produces the lowest EER and the highest $d^{\prime}$ for all experiments. For instance, for a time difference of 1 month the EER is $6.39 \%$ and the $d^{\prime}$ is 2.24. Furthermore, using a non-normalized version of the silhouettes improves performance. The GEnI uses a normalized silhouette and the EER are always higher and $d^{\prime}$ is always lower compared to all types of non-normalized GEI signatures. For instance, for a time difference of 1 month the EER is $14.57 \%$ and $d^{\prime}$ is 1.90 in the case of GEnI. Although the $d^{\prime}$ statistics ignores moments higher than second order it was considered adequate for all experiments in this study.

Fig. 5 shows that the ROC curves for all combinations of gallery and probe (shown in Table II) are very similar and overlapping. The curve that significantly differs from the rest is for a gallery and probe taken on the same day.

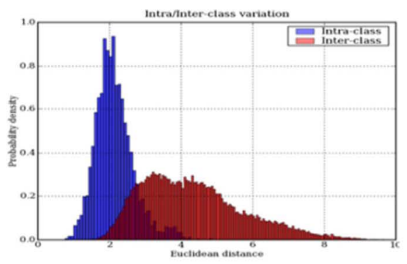

a)

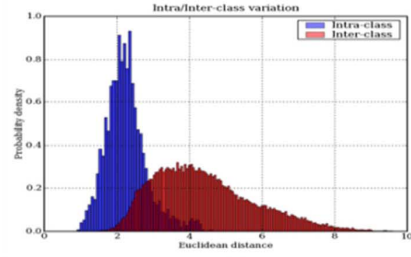

b)

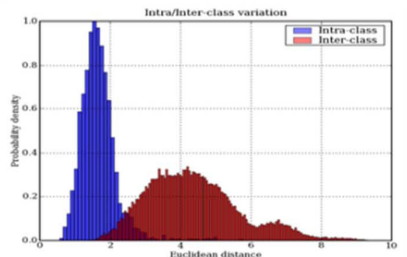

c)

Fig. 6. (a) Intra/inter class variation for time difference of 1 month, (b) time difference of 9 months and (c) time difference of few minutes.

Fig. 6 shows a set of intra/inter class variation diagrams. The Euclidian distance is plotted on the horizontal axis and the probability density on the vertical. The diagrams in Fig. 6(a) and (b) refer to different time periods and (c) refers to probe and gallery acquired within few minutes. The distributions in (a) and (b) are very similar. However, there is less overlap between the intra-class and inter-class distributions in (c).

There is only a $5 \%$ drop in performance over 9 months when the combination of side, front and top is used. Decidability decreases and EER increases for analysis over time compared to analysis on the same day. There is less overlap between the distributions in Fig. 6(c) and more area under the ROC curve for data captured on the same day. It is not clear whether the slight drop in performance over time can be attributed to time (aging) itself or to covariate factor(s) that we have yet to consider. However, our hypothesis is that the variation of performance over time can be caused by change of clothing underneath the overalls, as well as some change due to elapsed time. The best recognition is achieved when comparing similar temperature seasons (e.g., summer $\rightarrow$ spring). Presumably, subjects wore a similar style of clothes underneath the overalls. Having subjects wear a body suit (without clothes underneath) was considered but discarded as it was seen as impractical. 


\begin{tabular}{|c|c|c|c|c|c|}
\hline Dataset & MIT 2002 & HumanID & $\begin{array}{c}\text { Soton } \\
2002\end{array}$ & UMD & $\begin{array}{c}\text { Soton } \\
\text { Temporal }\end{array}$ \\
\hline CCR (\%) & 45 & 10 & 37 & 30 & $\mathbf{9 6}$ \\
\hline
\end{tabular}

Fig. 7. Highest CCR achieved by studies over time using various datasets.

The values of CCR, $\mathrm{d}^{\prime}$ and EER for the front and side views are similar over time. There is more dynamic gait information available when the side view is used, compared to the frontal view. Therefore, a question is raised how much of the dynamic information is used for recognition over time in the case of model-free approaches, such as the GEI and GEnI. Nevertheless, the results show that the top view is least suitable for recognition over time.

Achieving higher recognition rates and lower error rates could have been possible if more sophisticated classification techniques had been used. Nevertheless, the results presented in this section show that it is possible to recognize someone reliably by their gait after a certain time period has elapsed, if we were able to control several other essential parameters (like clothing and footwear). Although it is not possible to compare the results available in the literature due to significant difference in the datasets used, Fig. 7 provides a clear indication that "elapsed time" has been confused with other covariates so far in the literature. The conflating covariates have been the main reasons for low CCRs in the previous studies that have employed similar techniques to the ones used here.

\section{B. Effect of Clothes}

The main purpose of the experiments presented in this section is to provide an indication of why previous studies have achieved significantly lower recognition rates over time, by employing similar techniques to this study. The results could be improved if algorithms that are less sensitive to change in clothing are used. However, this paper does not focus on improving performance of recognition approaches but on understanding the effect of time on the performance of the baseline algorithm and a more recent gait representation.

Clothes can affect the overall body shape and certain types clothing can affect the way a person walks. Previous studies have indicated that change in clothing can have a negative effect on the performance of current gait matchers. A study [9] has reported a recognition rate of just $3 \%$ for the combination of the following covariate factors: clothes, shoes and time. Another study [7] reported a significant drop in performance $(87 \%$ to $60 \%$ ) when subjects wore trench coat on top of their normal clothes.

The data acquired in months 9 and 12 contain samples of subjects wearing different types of clothes and enables us to perform analysis of different type of clothes over time and over few minutes. It enables us to quantify the effect of clothes, while keeping all other covariates unchanged. There are four different types of clothes, three types of "ordinary" clothes of subject's choice and overalls provided as part of this experiment. Refer back to Section II for details. In this section, "Type I" clothes refer to the samples collected in month 9. "Type II" and "Type III" refer to the samples collected in month 12 .

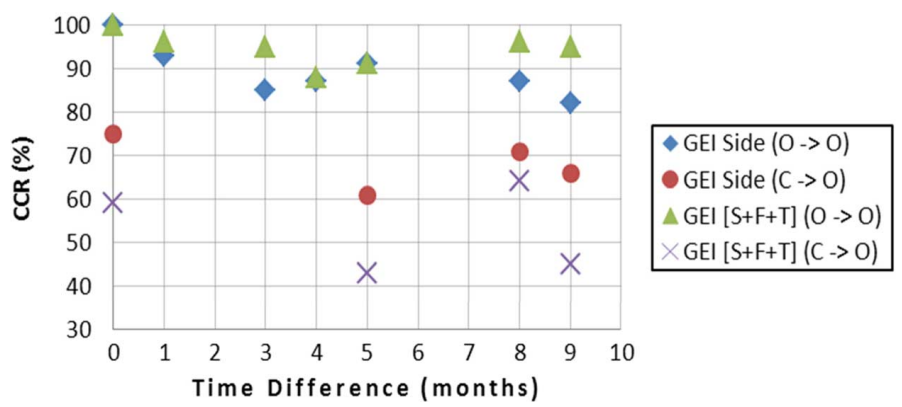

Fig. 8. The recognition performance over time. $(\mathrm{O} \rightarrow \mathrm{O}=$ Overall $\rightarrow$ Overall, $\mathrm{C} \rightarrow \mathrm{O}=$ Normal Clothes $\rightarrow$ Overall).

TABLE III

Combination of Probe ANd Gallery

\begin{tabular}{ccc}
\hline \hline $\begin{array}{c}\text { Probe } \\
\text { 10 samples/subject }\end{array}$ & $\begin{array}{c}\text { Gallery } \\
\text { 10 samples/subject }\end{array}$ & $\begin{array}{c}\text { Time Diff. } \\
\text { (months) }\end{array}$ \\
\hline Type I - 21 subj. & Month 9 Overall - 21 subj. & 0 \\
Type I - 21 subj. & Month 4 Overall - 22 subj. & 5 \\
Type I - 21 subj. & Month 1 Overall - 23 subj. & 8 \\
Type I -21 subj. & Month 0 Overall - 25 subj. & 9 \\
\hline \hline
\end{tabular}

Our clothing analysis consists of three experiments. In the first one we utilize the temporal data and perform matching over time with the subjects wearing different clothes. In experiment 2 we use the data acquired in month 9 to investigate the effects of clothing irrespectively of any other covariate, and finally in experiment 3 we use the data collected in month 12 to further quantify the effect as it provides additional clothing types.

Experiment 1: The purpose of this experiment is to perform matching of samples acquired at different time periods with subjects wearing different clothes. In addition to the combinations of gallery and probe shown in Table II the combinations shown in Table III are used. Two-fold cross validation is performed. Only the highest CCRs achieved are shown in Fig. 8. The highest value for the standard deviation of the CCR is 0.04 . The results presented in Fig. 8 indicate that different types of clothes can lead to a large drop in performance. The finding is similar for all signatures considered, but only the GEI (side) and $\mathrm{GEI}(\mathrm{S}+\mathrm{F}+\mathrm{T})$ are shown on the diagram for clarity. It is important to note that the drop in recognition is consistent with time.

Experiment 2: The aim of this experiment is to investigate the effect of different clothes in the case when the time between recording the gallery and the probe is few minutes and no other changes in the experimental condition occur. Table IV shows the combinations of probe and gallery used. Fig. 9 summarizes the results. The horizontal axis shows the combination of clothing considered. Type I clothes refer to the samples of subjects wearing "normal" clothes. Very high recognition results for all views are achieved if the same types of clothes are matched. However, when different types of clothes are used the recognition rates can fall to $40 \%$.

One ROC curve in Fig. 10 significantly differs from the others. Clothing change affects the curve's shape dramatically. The remainder of the curves represent samples of subjects wearing the same clothes but recorded in different sessions. 


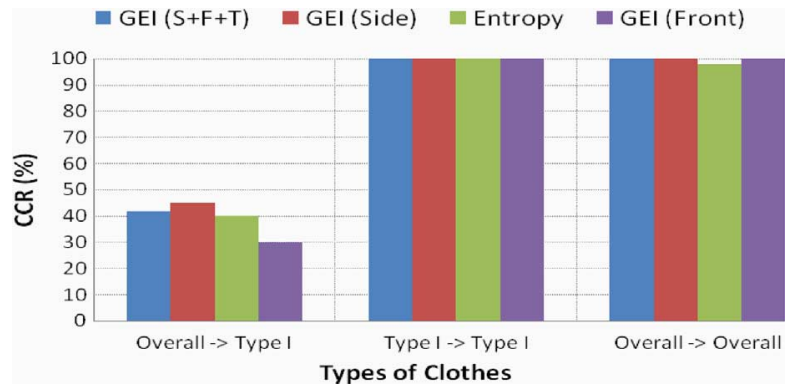

Fig. 9. Effect of clothes on the recognition performance. The probe and the gallery have been taken few minutes apart.

TABLE IV

COMBINATION OF Probe AND GaLlery

\begin{tabular}{ccc}
\hline \hline Probe & Gallery & Time \\
5 samples/subject & 5 samples $/$ subject & Diff. \\
\hline Overall -21 subj. & Type I -21 subj. & Few min. \\
Type I -21 subj. & Type I - 21 subj. & Few min. \\
Overall -21 subj. & Overall -21 subj. & Few min. \\
\hline \hline
\end{tabular}

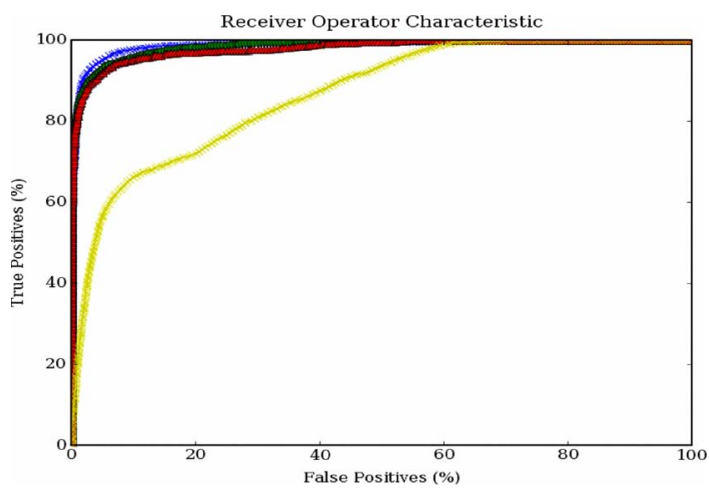

Fig. 10. ROC curve quantifying the effect of clothing.

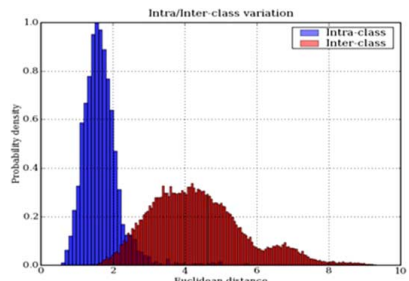

a)

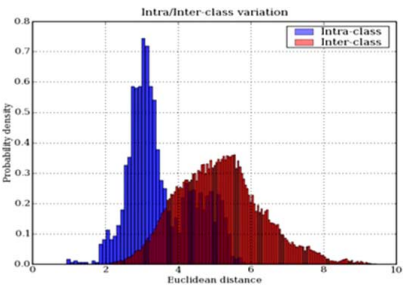

b)
Fig. 11. Inter/intra class variations for different types of clothes. (a) GEI (side)-Same clothes $\mathrm{d}^{\prime}=2.71, \mathrm{CCR}(\mathrm{k}=1)=99 \%, \mathrm{CCR}(\mathrm{k}=3)=$ $99 \%$, (b) GEI (side)-Different clothes $\mathrm{d}^{\prime}=1.57, \mathrm{CCR}(\mathrm{k}=1)=$ $44 \%, \mathrm{CCR}(\mathrm{k}=3)=45 \%$.

Fig. 11 shows the class distributions for the GEI (side) signature in the case of (a) same clothes and (b) different clothes.

The results demonstrate that high recognition rates can be achieved if clothes of the same type are matched and very low rates if "extreme" changes in clothing occur. The experiment described in part 3 investigates how the recognition rate is affected by change from one type of "ordinary" clothes to another, as opposed to "extreme" clothing variations such as the overall suit.

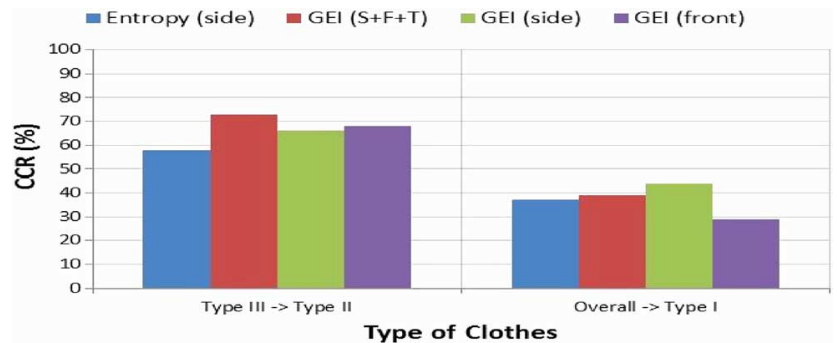

Fig. 12. Effect of different types of clothes on the recognition performance for various gait matchers.

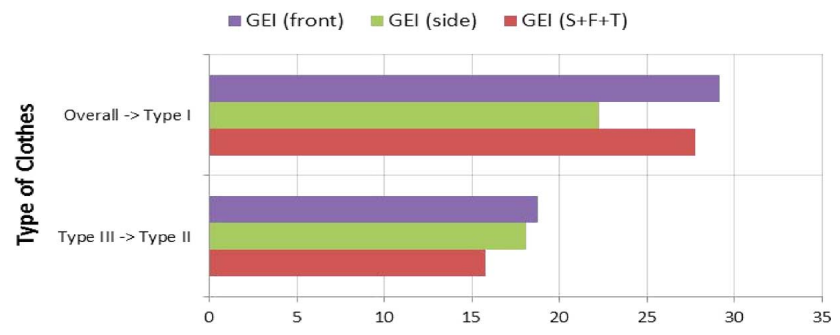

Fig. 13. EER for different types of clothes.

TABLE V

COMBINATION OF PROBE AND GALLERY

\begin{tabular}{ccc}
\hline \hline Probe & Gallery & Time \\
10 samples/subject & 10 samples/subject & Diff. \\
\hline Type III -18 subj. & Type II -18 subj. & Few min. \\
Overall -18 subj. & Type I -18 subj. & Few min. \\
\hline \hline
\end{tabular}

Experiment 3: We use the data captured in month 12 for this part of the experiment. Table $\mathrm{V}$ shows the combination of probe and gallery used. Fig. 12 summarizes the results. The EER for different gait signatures is shown in Fig. 13. It can be clearly seen that the EER increases if the subjects wear clothes that significantly differ from each other. The $d^{\prime}$ decreases in this case. The results of this experiment show that the recognition rates decrease significantly less if different but less extreme clothing changes occur.

In conclusion, the recognition can be affected significantly due to clothes change regardless of elapsed time or any other covariates. Furthermore, the drop in performance is less significant if subjects are wearing similar types of clothes. Another study [7] performed on a different database came to a similar conclusion for a model-based approach. The CCR dropped significantly when subjects wore trench coats. The results presented in this section are similar for the other studies that have considered the effect of elapsed time. This is an indication that elapsed time has been confused with other covariates (mainly clothing) so far in the literature.

\section{Footwear}

The combination of probe and gallery used is shown in Table VI. The data captured in month 12 is used. From Table VI, it can be concluded that wearing footwear is unlikely to drastically affect recognition. However, there is an indication 
TABLE VI

COMBINATION OF PROBE AND GALLERY

\begin{tabular}{ccc}
\hline \hline Probe & Gallery & Lowest/Highest \\
5 samples/subject & 5 samples/subject & CCR \\
\hline No shoes $-\mathbf{1 8}$ subj. & No shoes -18 subj. & $100 / 100$ \\
No shoes -18 subj. & With shoes -18 subj. & $97 / 100$ \\
\hline \hline
\end{tabular}

TABLE VII

COMBINATION OF PROBE AND GALLERY

\begin{tabular}{ccc}
\hline \hline Probe & Gallery & Relative Speed \\
5 samples/subject & 5 samples/subject & \\
\hline Probe 1 (22 subj.) & Gallery 1 (22 subj.) & Similar \\
Probe 2 (22 subj.) & Gallery 2 (22 subj.) & Different \\
\hline \hline
\end{tabular}

\section{Comparison of Equal Error Rates (EER)}

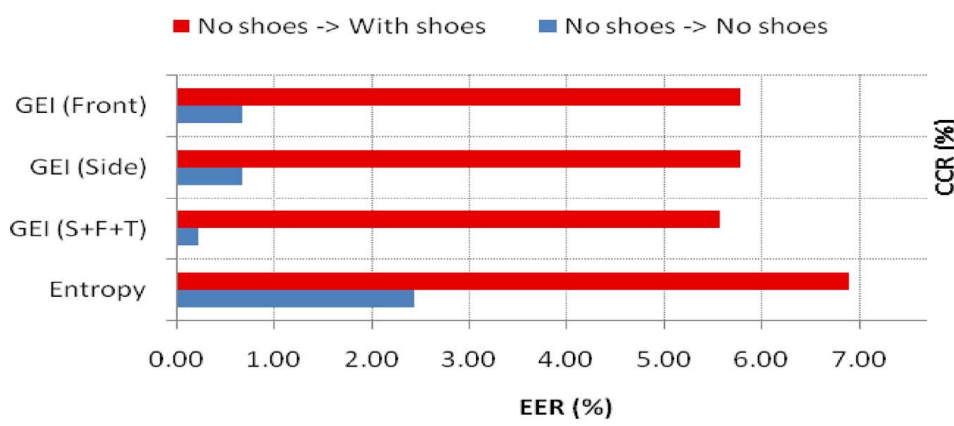

Fig. 14. EER for the footwear experiment.

that the EER can increase (Fig. 14). Further investigation using a larger dataset containing a larger variation of shoes is needed.

A study [7] employing a model-based approach reached similar result to ours. The CCR did not decrease dramatically when subjects' shoe type changed (except in the case of flip-flops). Another study [9] using the GEI representation has also achieved a relatively high recognition rate for a change in footwear.

\section{Speed}

A study [28] demonstrated the need for speed adjustment of gait features. A different study [7] showed that variation in walking speed can have a negative effect on the performance of model-based approaches. The recognition performance decreased from $87 \%$ to $60 \%$.

In this study, we use the combination of gallery and probe as shown in Table VII. Each gallery and probe consists of 110 samples and all samples are taken only few minutes apart. Other covariates remain unchanged. The walking speed was not controlled; we are just utilizing the natural variation in the subject's speed to perform this experiment. Each acquisition session consists of 20 samples per subject. For each subject, ten samples with similar walking speed were identified and five were added to "Gallery 1" and the other five to "Probe 1". In addition, ten samples of different walking speed were identified for each subject and five added to "Gallery 2" and the other five to "Probe 2 ". In this experiment we are interested in the relative change of walking speed between different samples of the same subject. The smallest variation in walking speed for a subject is $5.8 \%$ and the greatest variation for a particular subject is $25 \%$.

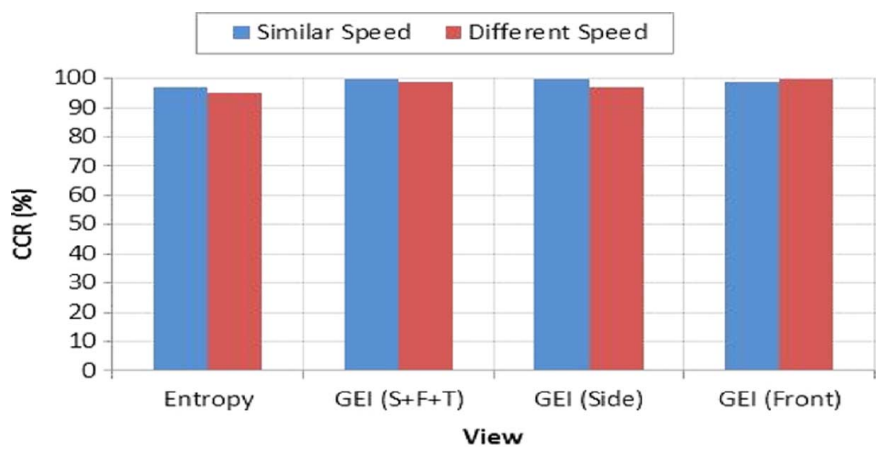

Fig. 15. Results of the walking speed experiment.

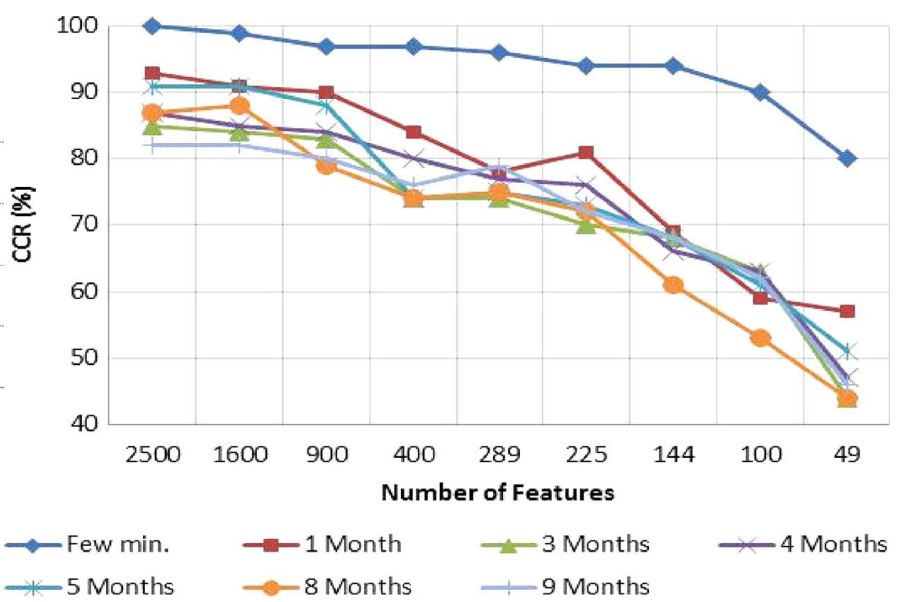

Fig. 16. Number of features versus Performance, over time. Time periods refer to the elapsed time between capturing the gallery and the probe.

This data applies for the acquisition session taken in "month 1". However, it is anticipated that the variations are similar for the other sessions because there were no changes in the experimental conditions. The results are presented in Fig. 15. There is a slight drop in performance for all signatures, except for GEI (Front). It would appear that the side view is mostly affected by change of speed which is somewhat expected.

Recently, Aqmar et al. [29] proposed a gait representation that is robust to variations in speed.

\section{E. Number of Features Versus Performance Over Time}

A unique advantage of gait is the capability to do recognition at a distance, at a low resolution. Therefore, it is important to show how the performance changes with number of features. In this study, the number of features equates to the number of pixels in the GEI or GEnI signature. If gait is captured at a distance there are fewer pixels in the image that can be used for recognition. Consequently, a decrease in the number of features is equivalent to an increase in the distance at which the subject is observed. The rate at which the performance deteriorates with smaller number of features should be consistent over time. In other words, if the gallery and probe are acquired $m$ month apart and a recognition performance of $p$ is achieved with $n$ number of features then a $p \pm q$ (where $q$ is very small) performance for the same number of features $n$ should be expected if the gallery and probe are acquired $m+o$ months apart ( $o$ any value). 
We have performed an experiment using GEI (side view) to show whether time has any effect on the ability to do gait recognition at a lower resolution. The findings are presented in Fig. 16. We have shown that irrespective of the time difference (except when the time difference is few minutes) between the probe and the gallery the degradation of performance as the number of features decrease is fairly consistent. This is evidence that gait recognition can be used as a reliable biometrics at a distance over time. Fig. 16 shows that high performance can be achieved by using a small number of features. Using only 900 features $(30 \times 30)$ a CCR of $80 \%-90 \%$ can be achieved. However, a larger database is needed to correctly verify the number of features $n$ needed to achieve a recognition $p$. There is ongoing work in developing gait recognition algorithms capable of achieving high recognition rates at low resolutions [30]. The main aim of this experiment is to show that elapsed time does not play an important role to achieve recognition at various resolutions.

\section{CONCLUSION}

In studies to date, there has been a lack of understanding of elapsed time on the performance of gait recognition. The goal of this paper was to study the effect of time on gait recognition by controlling all covariate factors and removing their influence. This study analyses various covariate factors using existing approaches to provide an indication of why previous studies have achieved low recognition rates over time. The major obstacle for quantifying the effect of time and other factors independently is the lack of suitable databases and conflating more than one covariate between different acquisition sessions. We have created a novel multimodal temporal database which could be used for developing and evaluating covariate invariant algorithms. It is worth noting that the paper does not aim at proposing new recognition algorithms.

We have shown for the first time that gait per se is time-invariant in the short and short/medium term and, thus, can be used as a reliable biometric trait over time if influential covariant factors (namely clothing, footwear) were controllable. A similar recognition performance was achieved over seven different time periods and a CCR of $95 \%$ is achieved over period of 9 months. We hypothesize that a CCR of nearly $100 \%$ could be achieved if various covariate factors (like clothing, footwear etc.) were controllable. Unfortunately, this situation is unlikely to occur in the real world. We have shown that the major problem for recognition over time can be partially attributed to the change of clothes. We have shown a dramatic fall in performance in the case of "extreme" changes in clothes. However, given that the problem of clothing can be overcome using techniques as in [25], [31], the issue of gaining/losing weight would still pose a challenge in the case of model-free approaches, such as the GEI. Further research is needed to identify an age-invariant feature subspace for gait by performing feature set selection [25], [32].

A second significant finding is that we have shown that other authors reported low gait recognition since clothing and other covariates have been confused with "elapsed time" previously in the literature. The CCR drops from $100 \%$ to $30 \%$ for nontemporal combination of gallery and probe, which corresponds to the results achieved by other studies over time.
We have also confirmed that recognition by gait at low resolution (at a distance) is not affected by elapsed time. This is a very significant finding because recognition at a distance is a major advantage of gait over all other biometrics.

It would appear that shoes and speed are unlikely to affect recognition performance significantly in the case of model-free gait representations. However, it is important to note that the data collected to analyze the effect of these covariate factors can merely provide indication and not quantify the exact effect.

Experiments performed outdoors were not considered appropriate for this study because additional environment related covariates would be introduced and it would be difficult to quantify how much recognition changes due to the imperfections of the extracted silhouettes and how much due to elapsed time or other subject specific covariates.

Both approaches used in this work are model-free and therefore the conclusions in this study should be verified for modelbased algorithms. However, our results show that recognition is possible over a significant period of time and at a low resolution. These results bring gait one step closer to being used as a forensic tool.

\section{REFERENCES}

[1] Z. Xue, D. Ming, W. Song, B. Wan, and S. Jin, "Infrared gait recognition based on wavelet transform and support vector machine," Pattern Recognition, vol. 43, no. 8, pp. 2904-2910, 2010.

[2] K. Bashir, T. Xiang, and S. Gong, "Gait recognition without subject cooperation," Pattern Recognition Lett., vol. 31, no. 13, pp. 2052-2060, 2010.

[3] F. Jean, A. B. Albu, and R. Bergevin, "Towards view-invariant gait modeling: Computing view-normalized body part trajectories," Pattern Recognition, vol. 42, no. 11, pp. 2936-2949, 2009.

[4] I. Bouchrika, M. Goffredo, J. N. Carter, and M. S. Nixon, "On using gait in forensic biometrics," J. Forensic Sci., vol. 56, no. 4, pp. 882-889, 2011.

[5] P. K. Larsen, E. B. Simonsen, and N. Lynnerup, "Gait analysis in forensic medicine," J. Forensic Sci., vol. 53, no. 5, pp. 1149-1153, 2008.

[6] I. Bouchrika, M. Goffredo, J. Carter, and M. Nixon, "Covariate analysis for view-point independent gait recognition," in Proc. 3rd IAPR Int. Conf. Biometrics, Italy, 2009.

[7] I. Bouchrika and M. Nixon, "Exploratory factor analysis of gait recognition," in Proc. 8th IEEE Int. Conf. Automatic Face and Gesture Recognition (AFGR), Amsterdam, The Netherlands, 2008.

[8] Y. Shiqi, T. Daoliang, and T. Tan, "A framework for evaluating the effect of view angle, clothing and carrying condition on gait recognition," in Proc. 18th Int. Conf. Pattern Recognition (ICPR), 2006, pp. 441-444.

[9] S. Sarkar, P. J. Phillips, L. Zongyi, V. I. Robledo, P. Grother, and K. W. Bowyer, "The HumanID gait challenge problem: Data sets, performance, and analysis," IEEE Trans. Pattern Analysis Machine Intell., vol. 27 , no. 2 , pp. $162-177$, Feb. 2005.

[10] L. Lee and W. E. L. Grimson, "Gait analysis for recognition and classification," in Proc. 5th IEEE Int. Conf. AFGR, 2002, pp. 148-155.

[11] B. Bhanur and J. Han, "Model-based human recognition-2D and 3D gait," in Human Recognition at a Distance in Video. London, U.K.: Springer, 2011, pp. 65-94, Advances in Pattern Recognition.

[12] G. Junxia, D. Xiaoqing, W. Shengjin, and W. Youshou, "Action and gait recognition from recovered 3-D human joints," IEEE Trans. Syst., Man, Cybern., Part B: Cybernetics, vol. 40, no. 4, pp. 1021-1033, Apr. 2010.

[13] J. Han and B. Bhanu, "Individual recognition using gait energy image," IEEE Trans. Pattern Anal. Machine Intell., vol. 28, no. 2, pp. 316-322, Feb. 2006.

[14] C. Chen, J. Liang, H. Zhao, H. Hu, and J. Tian, "Frame difference energy image for gait recognition with incomplete silhouettes," Pattern Recognition Lett., vol. 30, no. 11, pp. 977-984, 2009.

[15] Z. Liu and S. Sarkar, "Simplest representation yet for gait recognition Averaged silhouette," in Proc. 17th IEEE Int. Conf. Pattern Recognition (ICPR), Cambridge, U.K., 2004, pp. 211-214. 
[16] K. Bashir, T. Xiang, and S. Gong, "Gait recognition using gait entropy image," in Proc. 3rd Int. Conf. Crime Detection and Prevention (ICDP 2009), 2009, pp. 1-6.

[17] G. Veres, M. Nixon, and J. Carter, "Model-based approaches for predicting gait changes over time," in Proc. Int. Conf. ISSNIP, 2005, pp. 325-330.

[18] Z. Liu, L. Malave, and S. Sarkar, "Studies on silhouette quality and gait recognition," in IEEE Int. Conf. CVPR, Washington, D.C., 2004, pp. 704-711.

[19] R. T. Collins, R. Gross, and J. Shi, "Silhouette-based human identification from body shape and gait," in Proc. 5th IEEE Int. Conf. AFGR, 2002, pp. 366-371.

[20] A. Kale, A. Rajagopalan, N. Cuntoor, and V. Kruger, "Gait-based recognition of humans using continuous HMMs," in Proc. 5th IEEE Int. Conf. AFGR, 2002, pp. 336-343.

[21] M. Okumura, H. Iwama, Y. Makihara, and Y. Yagi, "Performance evaluation of vision-based gait recognition using a very large-scale gait database," in Proc. 4th IEEE Int. Conf. Biometrics: Theory, Applications and Systems (BTAS), Washington, D.C, 2010.

[22] D. Tao, X. Li, X. Wu, and S. Maybank, "Elapsed time in human gait recognition: A new approach," in Proc. IEEE Int. Conf. ICASSP, 2006.

[23] D. S. Matovski, M. S. Nixon, S. Mahmoodi, and J. N. Carter, "The effect of time on the performance of gait biometrics," in Proc. 4th IEEE Int. Conf. Biometrics: Theory, Applications and Systems (BTAS), Washington, D.C., 2010.

[24] M. A. Hossain, Y. Makihara, W. Junqui, and Y. Yagi, "Clothing-invariant gait identification using part-based clothing categorization and adaptive weight control," Pattern Recognition, vol. 43, no. 6, pp. 2281-2291, 2010.

[25] I. Venkat and P. De Wilde, "Robust gait recognition by learning and exploiting sub-gait characteristics," Int. J. Computer Vision, vol. 91, no. 1, pp. 7-23, 2011.

[26] S. Samangooei, J. Bustard, M. S. Nixon, and J. N. N. Carter, On Acquisition and Analysis of a Dataset Comprising of Gait, Ear and Semantic Data. In Multibiometrics for Human Identification. Cambridge, U.K.: Cambridge Univ. Press, 2010.

[27] J. Daugman, Biometric Decision Landscapes Univ. Cambridge Computer Lab., Tech. Rep., 2000.

[28] R. Tanawongsuwan and A. Bobick, "Performance analysis of time-distance gait parameters under different speeds," Lecture Notes in Computer Sci., pp. 715-724, 2003.

[29] M. R. Aqmar, K. Shinoda, and S. Furui, "Robust gait recognition against speed variation," in Proc. 20th IEEE Int. Conf. Pattern Recognition (ICPR), Istanbul, Turkey, 2010.

[30] Z. Junping, P. Jian, C. Changyou, and R. Fleischer, "Low-resolution gait recognition," IEEE Trans. Syst., Man, Cybern., Part B: Cybernetics, vol. 40, no. 4, pp. 986-996, Apr. 2010.

[31] A. Balan and M. Black, "The naked truth: Estimating body shape under clothing," in Proc. ECCU, 2008, pp. 15-29.

[32] B. Guo and M. Nixon, "Gait feature subset selection by mutual information," IEEE Trans. Syst., Man, Cybern., Part A: Systems and Humans, vol. 39, no. 1, pp. 36-46, Jan. 2009.

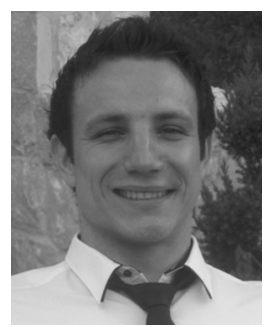

Darko S. Matovski received the B.Eng. degree in computer engineering from the School of Electronics and Computer Science, University of Southampton, U.K. He is working towards the M.B.A. and Engineering Doctorate degrees from the same university in the area of gait recognition.

$\mathrm{He}$ has also been working as a Research Engineer at the U.K, National Physical Laboratory on projects related to translating gait recognition research to real-life environments. His research interests are: image processing, computer vision, gait biometrics and visual surveillance.

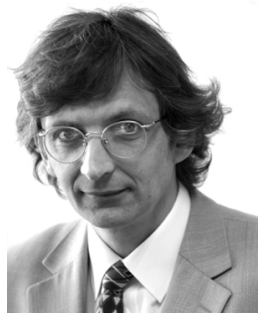

Mark S. Nixon is a Professor in computer vision at the University of Southampton, Southampton, U.K His research interests are in image processing and computer vision. His team develops new techniques for static and moving shape extraction which have found application in automatic face and automatic gait recognition and in medical image analysis. His team contains early workers in face recognition, and they later came to pioneer gait recognition and more recently joined the pioneers of ear biometrics. His vision textbook, with A. Aguado, Feature Extraction and Image Processing (Academic) reached second edition in 2008. With T. Tan and R. Chellappa, the book Human ID Based on Gait is part of the Springer Series on Biometrics and was published in 2005.

Dr. Nixon has chaired or had major involvement in many conferences (BMVC, AVBPA, IEEE Face and Gesture, ICPR, ICB, IEEE BTAS) and has given many invited talks. He is a Fellow IET and FIAPR.

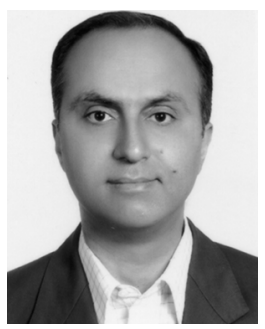

Sasan Mahmoodi (M'02) received the B.Sc. degree from Amirkabir University of Technology in 1988 the M.Sc. degree from Sharif University of Technology, Tehran, Iran, in 1991, and the Ph.D. degree from the University of Newcastle, Newcastle upon Tyne, U.K., in 1998.

In 1999, he held a Research Fellowship post in mathematical analysis of human vision in the University of Newcastle upon Tyne. He joined the University of Southampton in 2007 as a Lecturer. His current research interests are in image processing, com-

puter vision and Biometrics.

Dr. Mahmoodi sits in the executive committee of BMVA.

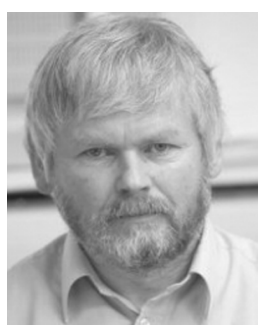

John N. Carter (M'90) received the B.A. degree in experimental physics from Trinity College, Dublin, Ireland, and the Ph.D. degree in astrophysics from the University of Southampton, Southampton, U.K.

In 1985, he changed discipline and joined the School of Electronics and Computer Science, University of Southampton, as a Lecturer researching in signal and image processing, where he is currently a Senior Lecturer with the Information: Signals, Images and Systems Research Group. In the past, he worked on programs as diverse as diesel engine diagnostics and vocal tract imaging. A recent success in this field is the development of a new dynamic form of magnetic resonance imaging, which makes it possible to reconstruct high time resolution multiplanar views of the human vocal tract while a subject is repeating a short phrase. His current research interest is in the general area of 4-D image processing, i.e., analyzing sequences of images to extract both 2-D and 3-D features, exploiting coherence over the whole sequence, i.e., imposing simple smoothness and continuity constraints. This has applications in object tracking and feature detection, where it is tolerant of high levels of noise and missing data. This has found application in biometrics, particularly in automatic gait analysis. 